\title{
Experiments and multiscale simulations of laser propagation through ignition-scale plasmas
}

\author{
S. H. GLENZER ${ }^{1 *}$, D. H. FROULA ${ }^{1}$, L. DIVOL ${ }^{1}$, M. DORR ${ }^{1}$, R. L. BERGER ${ }^{1}$, S. DIXIT ${ }^{1}$, B. A. HAMMEL ${ }^{1}$, \\ C. HAYNAM ${ }^{1}$, J. A. HITTINGER ${ }^{1}$, J. P. HOLDER ${ }^{1}$, 0. S. JONES ${ }^{1}$, D. H. KALANTAR ${ }^{1}$, 0. L. LANDEN ${ }^{1}$, \\ A. B. LANGDON1, S. LANGER¹, B. J. MACGOWAN11, A. J. MACKINNON11, N. MEEZAN1, E. I. MOSES1, \\ C. NIEMANN ${ }^{1,2}$, C. H. STILL1 ${ }^{1}$, L. J. SUTER ${ }^{1}$, R. J. WALLACE1 ${ }^{1}$, E. A. WILLIAMS ${ }^{1}$ AND B. K. F. YOUNG ${ }^{1}$ \\ ${ }^{1}$ Lawrence Livermore National Laboratory, L-399, P0 Box 808, Livermore, California 94551, USA \\ ${ }^{2}$ University of California Los Angeles, Electrical Engineering Department, Los Angeles, California 90095, USA \\ *e-mail: glenzer1@Inl.gov
}

With the next generation of high-power laser facilities for inertial fusion coming online $\mathrm{e}^{1,2}$, ensuring laser beam propagation through centimetre-scale plasmas is a key physics issue for reaching ignition. Existing experimental results ${ }^{3-5}$ including the most recent one ${ }^{6}$ are limited to small laser spots, low-interaction laser beam energies and small plasma volumes of 1-2 mm. Here, we demonstrate the propagation of an intense, highenergy, ignition-size laser beam through fusion-size plasmas on the National Ignition Facility (NIF) and find the experimental measurements to agree with full-scale modelling. Previous attempts to apply computer modelling as a predictive capability have been limited by the inherently multiscale description of the full laser-plasma interaction processes ${ }^{7-11}$. The findings of this study validate supercomputer modelling as an essential tool for the design of future ignition experiments.

The National Ignition Facility (NIF) at the Lawrence Livermore National Laboratory in the USA $^{1}$ and the Laser Mega Joule in France $^{2}$ are being constructed with the goal to ignite deuteriumtritium plasmas by inertial confinement fusion ${ }^{12,13}$. In the indirectdrive approach to inertial confinement fusion, 192 laser beams (or 240 beams in the case of the Laser Mega Joule) irradiate the interior of a cylindrical high-Z cavity, called a hohlraum, with a fusion capsule placed in its centre. When the laser beams interact with the high- $Z$ wall, soft $X$-rays are produced that compress the spherical fusion capsule of $\sim 2 \mathrm{~mm}$ initial diameter to $1 / 35$ th of that size. This compression heats the deuterium-tritium fill to temperatures sufficient for ignition and burn. This process will create a microscopic star in the laboratory, the ultimate goal for studies of burning-plasma processes and astrophysical phenomena.

The laser-plasma interactions in these ignition hohlraums are complex because the laser beams will need to propagate through a large volume of plasma before they reach the high- $Z$ walls of the hohlraum. The total laser propagation length through the hohlraum plasma approaches $7 \mathrm{~mm}$ with electron temperatures in the range of $T_{\mathrm{e}}=3-5 \mathrm{keV}$ and electron densities of $n_{\mathrm{e}} \leq 10^{21} \mathrm{~cm}^{-3}$ (ref. 3). The laser beams must propagate through these plasmas to properly launch shocks into the fusion capsule, and to symmetrically implode the capsule to ignition conditions.
Our experiments use the first four beams, a quad of the NIF ${ }^{14-16}$, and show that properly smoothed laser beams propagate through the full length of fusion-size plasmas. Recent laser performance data ${ }^{17}$ from this laser show that the laser meets the original design projections required for ignition experiments. The experiments apply smoothing by spectral dispersion $(S S D)^{18}$ and polarization smoothing ${ }^{19,20}$ in addition to continuous-phase plates $(\mathrm{CPPs})^{21,22}$. These techniques greatly reduce the number of high-intensity speckles in the laser beam. SSD provides temporal beam smoothing and polarization smoothing provides a polarization-dependent instantaneous speckle intensity reduction, both efficiently suppressing the filamentation and self-focusing instability. Without SSD and polarization-smoothing laser-conditioning techniques, the experiments show that the laser beams filament in the plasma resulting in whole beam self-focusing and beam spray. These processes stall the propagation of the beams and delay the burn-through time from $1.5 \mathrm{~ns}$ to the end of the 3.5-ns-long laser pulse.

The experiment has been successfully modelled using massively parallel supercomputer calculations with the laser-plasma interaction code $\mathrm{pF} 3 \mathrm{D}^{10,11}$. These integrated calculations use, for the first time, realistic beams including micrometre-scale speckles and ponderomotive effects over the full $7 \mathrm{~mm}$ plasma length and for the entire $3.5 \mathrm{~ns}$ duration of the experiment. This was made possible by using advanced multiscale algorithms for solving the classical nonlinear heat-conduction equation ${ }^{23}$ on such a large computing mesh and efficient parallel computing techniques for solving hydrodynamics and the laser paraxial propagation. The simulations use three-dimensional (3D) classical heat transport and a 2D coupled-wave model of laser-plasma instabilities coupled with a nonlinear hydrodynamic module and rotated along the $z$ axis. A full run took two weeks on 512 processors.

In this experiment, the first four beams of the NIF (Fig. 1) have been focused into the centre of a $7-\mathrm{mm}$-long gas pipe target that was filled with $1 \mathrm{~atm}$ of $\mathrm{CO}_{2}$. The four beams heat the gas from one side and propagate along the axis of the target, overlapping in the centre with an effective $f / 8$ beam cone and a best (vacuum) focal spot diameter of $0.5 \mathrm{~mm}$. The total (vacuum) 


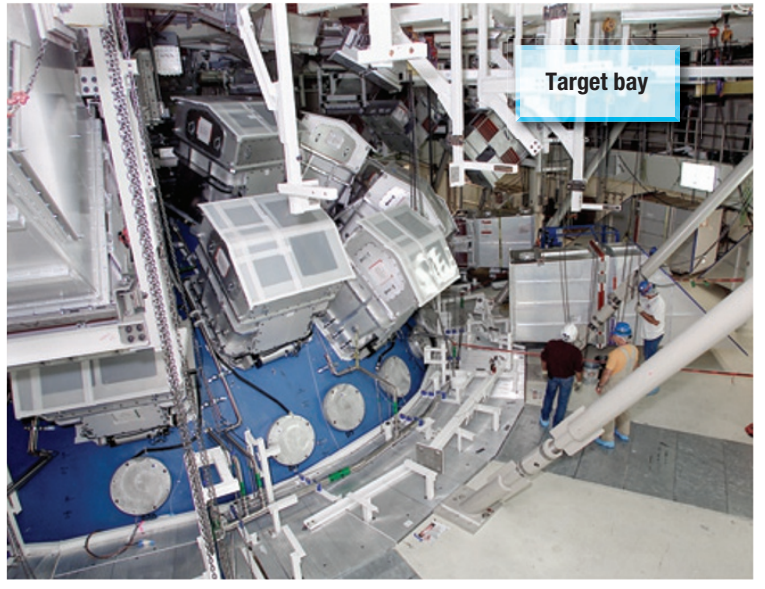

Figure 1 The National Ignition Facility's target bay. The present experiments have been carried out with four beams, frequency tripled to $351 \mathrm{~nm}$ and focused into the target chamber. Several beam ports of the remaining 188 beams that will be activated for ignition experiments by the end of this decade are also shown.

intensity of $I_{0}=2 \times 10^{15} \mathrm{~W} \mathrm{~cm}^{-2}$ was achieved where the four beams overlap with a total energy of $16 \mathrm{~kJ}$ in a 3.5 -ns-long square pulse. These irradiation conditions result in a large-scale plasma with temperatures approaching $T_{\mathrm{e}}=2 \mathrm{keV}$ and electron densities of $n_{\mathrm{e}}=6 \times 10^{20} \mathrm{~cm}^{-3}$ (ref. 14).

Figure 2 shows a schematic diagram of the experiment together with an image of the target and the X-ray emission data from two shots. The interaction of the laser beams with the target has been measured with the temporally resolved pinhole-based flexible $\mathrm{X}$-ray imager, and with laser backscattering diagnostics. The flexible $\mathrm{X}$-ray imager ${ }^{24}$ was filtered for $3.5 \mathrm{keV} \mathrm{X}$-rays and imaged the target with $\mathrm{a} \times 1$ magnification through multiple pinholes onto four gated strips of a microchannel plate detector. The strips were timed independently providing 2D snapshots with a temporal resolution of $\Delta t \sim 250 \mathrm{ps}$ and a spatial resolution of $200 \mu \mathrm{m}$. These laser-generated X-rays provide a signature of the laser-generated heat wave propagating through the target.

Consistent with the X-ray imaging data we find that measurements of the laser backscatter by the stimulated Brillouin scattering (SBS) and stimulated Raman scattering (SRS) processes provide evidence that filamentation is suppressed for fully smoothed beams. These measurements were carried out with the full-aperture backscatter station (FABS) ${ }^{25}$ and the near-backscatter imager $(\mathrm{NBI})^{26}$. The FABS consists of multiple detector systems measuring the scattered light from the target through the final focusing optics for each of the four beams. The optics and the final turning mirror transmit light backscattered from the target by SBS (between 351 and $352 \mathrm{~nm}$ ), as well as by SRS (between 450 and $700 \mathrm{~nm}$ ).

Complementing the FABS, the NBI measures SBS and SRS light that illuminates an absolutely calibrated scatter plate mounted inside the target chamber and around the openings of the four beams. Thus, with the exception of a small gap between the NBI plate and the FABS aperture, the total backscattered light by parametric laser-plasma instabilities is measured in these experiments. We find that the backscatter is dominated by SBS, with SRS being negligible. Moreover, the fraction of light scattered outside the initial $f$-cone and measured with the NBI cameras provides a signature of laser beam filamentation ${ }^{10}$.

When lasers interact with plasmas, electron density fluctuations refract the light towards lower-density plasma regions increasing

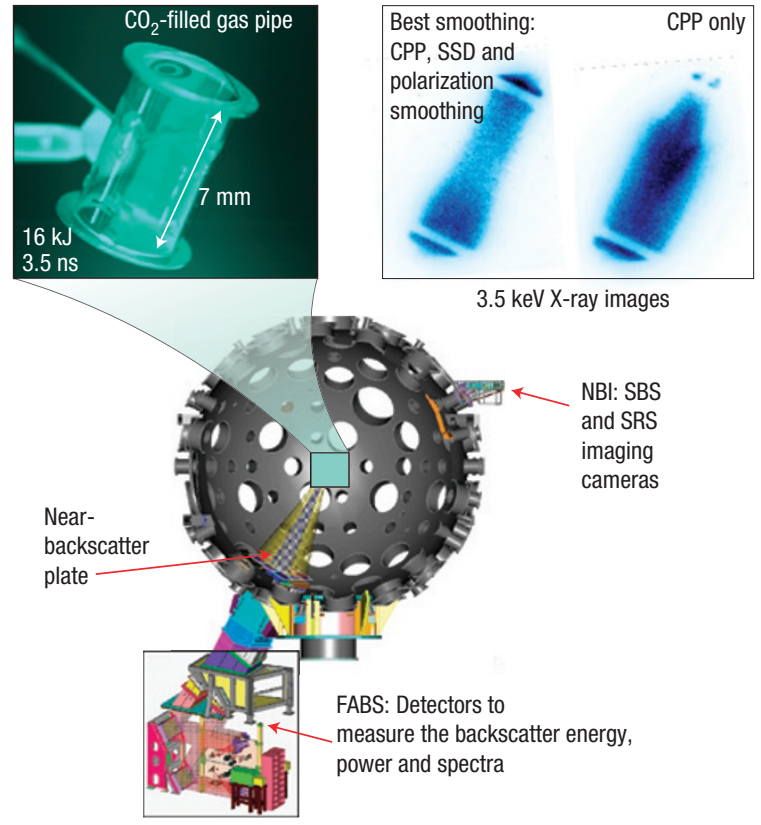

Figure 2 Schematic diagram of the laser beam propagation experiment on the NIF. The $3.5 \mathrm{keV} X$-ray image for the experiment with best smoothing was taken at $t=3.7 \mathrm{~ns}$ and shows full propagation. The experiment that only used CPP shows self-focusing and filamentation at $t=3.3 \mathrm{~ns}$.

locally the laser intensity. Filamentation occurs when the increased electric field of the laser drives plasma away, increasing the density depression, enhancing beam refraction and further increasing the local laser intensity. Consequently, the angular spread of the laser backscattering from a filamenting laser will be increased by refraction and will be larger than the initial laser $f$-cone.

Figure 3 shows measured and simulated SBS data on the NBI scatter plate. It is clear that the angular spread and the total amount of SBS on the NBI plate are reduced with full laser beam smoothing. For the case with beams smoothed only by CCP, we find that $25 \%$ of the total SBS is directly backscattered and detected by FABS, whereas with full beam smoothing (CPPs, SSD and polarization smoothing), $57 \%$ of the total SBS is detected by FABS. The relative reduction in spray of the backscattered light agrees with our modelling using the code $\mathrm{pF} 3 \mathrm{D}$ (described below). The calculations show a reduction in spray due to suppression of filamentation with full smoothing but slightly overestimate the total width of the intensity profile on the NBI plate.

Full smoothing also results in a slight reduction of the total SBS reflectivity; the time-integrated value is reduced from $41 \%$ to $36 \%$. The backscatter measurements are consistent with the $\mathrm{pF} 3 \mathrm{D}$ simulations that show SBS backscattering in the range of $30-50 \%$ at various times during the interaction. Both significant Landau damping of Langmuir waves in this low-density, relatively hightemperature plasma, coupled with longitudinal density gradients generated by the laser beam propagation contribute to low SRS. On the other hand, mid-Z atomic species result in weak Landau damping for ion acoustic waves and the linear gain for SBS is in the range 100-300. These conditions lead to pump depletion, consistent with the large backscatter measured and the relative ineffectiveness of SSD in reducing it. In this regime dominated by pump depletion, the calculated SBS reflectivity is not significantly affected by further nonlinear processes ${ }^{27-29}$, thus pF3D successfully calculates backscattering and filamentation. 


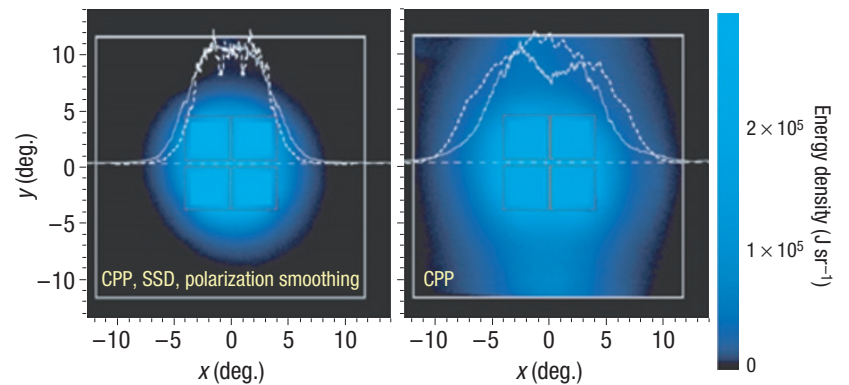

Figure 3 Measured and simulated NBI SBS data. Experiments with full smoothing show less light and reduced spray on the NBI plate. The width of the measured intensity profile at $y=0$ (solid line) is reduced by $15 \%$ with full smoothing consistent with modelling using the code pF3D (dashed line) that shows a $19 \%$ reduction due to suppression of the filamentation instability by beam smoothing.

Figure 4 shows experimental and calculated side-on X-ray images at various times during the experiment. With full smoothing we observe burn-through at $t=1.5 \mathrm{~ns}$ where the membrane at the end of the gas pipe begins to emit $3.5 \mathrm{keV} \mathrm{X}$-rays. This time is reproduced by the laser-plasma interaction calculations to within $100-200 \mathrm{ps}$, which is required for properly timing the shocks launched into the fusion capsule (ref. 12, page 12).

Full-scale pF3D calculations have successfully simulated the extent of the lateral and transverse heat wave inferred from the X-ray emission. With full smoothing, the modelling shows propagation determined by refraction and collisional absorption (top two rows of Fig. 4). The simulations correctly calculate beam spray due to small-scale laser beam speckles inaccessible to ray tracing. As a result, the modelling reproduces the stalled propagation for CPP only at $1.5 \mathrm{~ns}$ (bottom two rows of Fig. 4) and the overall shape of the X-ray emission.

A simple energy flux balance can help understand the laser burn-through process and its stalling when filamentation occurs. Equating the thermal energy evolution in the heated plasma region with inverse Bremsstrahlung heating from the laser beam of power $P$ (eventually reduced by backscatter losses), and lateral losses through electron heat conduction, $q$,

$$
\frac{3}{2} n_{\mathrm{e}} \frac{\partial}{\partial t} T_{\mathrm{e}}=-\frac{\partial}{\partial z} \frac{P}{A}-\nabla q
$$

Assuming steady state and integrating equation (1) over the laser beam area, $A=\pi r_{\mathrm{b}}^{2}$, and from $z=0$ to the heat front, $z_{\mathrm{f}}$, we find for $z_{\mathrm{f}} \gg r_{\mathrm{b}}$

$$
z_{\mathrm{f}}=\sqrt{\frac{P_{0}}{2 \pi r_{\mathrm{b}} \kappa^{*} q^{*}}} .
$$

Here the free-streaming heat flux, $q^{*}=n_{\mathrm{e}} v_{\mathrm{e}} T_{\mathrm{e}}^{*}$ and the inverse Bremsstrahlung absorption length, $\kappa^{*}$, is calculated at $T_{\mathrm{e}}^{*}=1 \mathrm{keV}$, where $v_{\mathrm{e}}$ is the thermal electron velocity. Equation (2) results in a heat-front distance, $z_{\mathrm{f}}$, consistent with the target length of $7 \mathrm{~mm}$. It further shows that increasing the laser beam radius by filamentation processes will stall propagation and reduce the beam propagation distance. This result is consistent with the measured laser beam propagation over the full scale of fusion-size plasmas due to the suppression of the filamentation instability.

This first truly ignition-scale laser-plasma interaction experiment on the NIF provided unambiguous data on the effect of
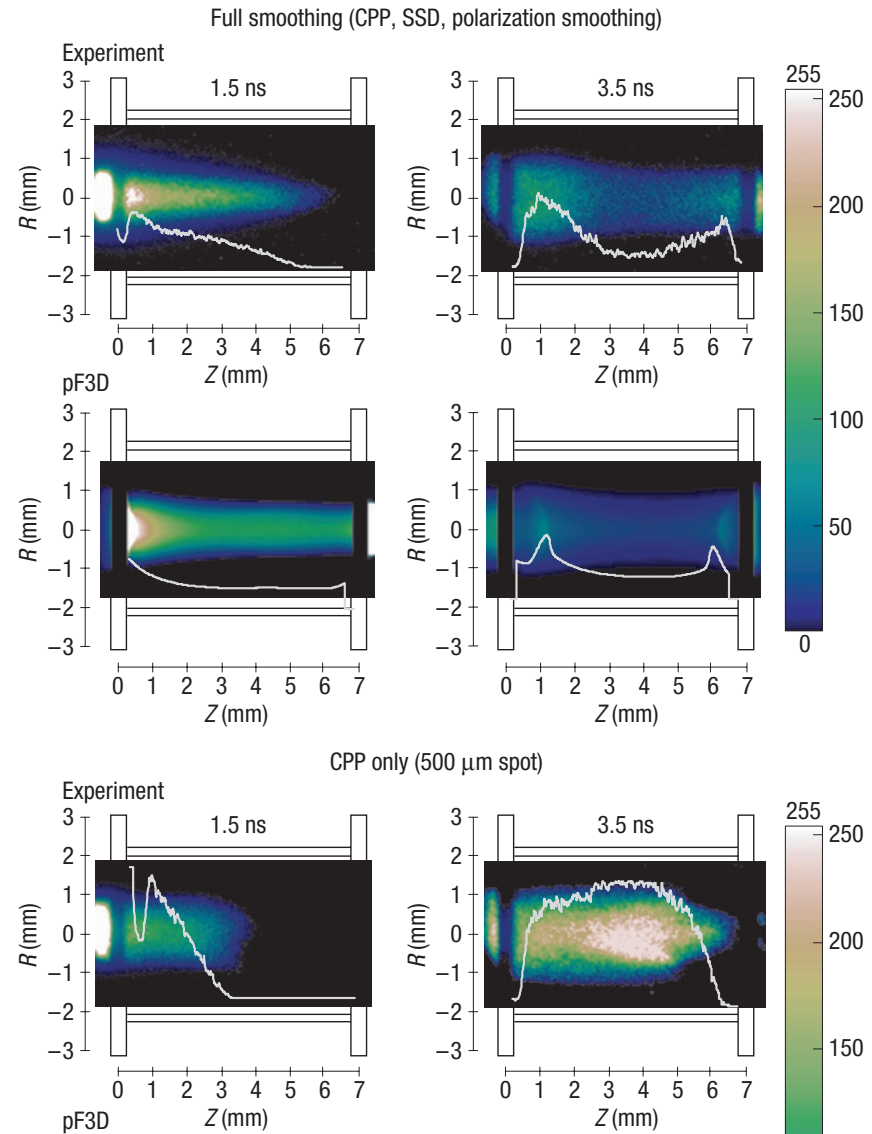

PP only (500 $\mu \mathrm{m}$ spot)
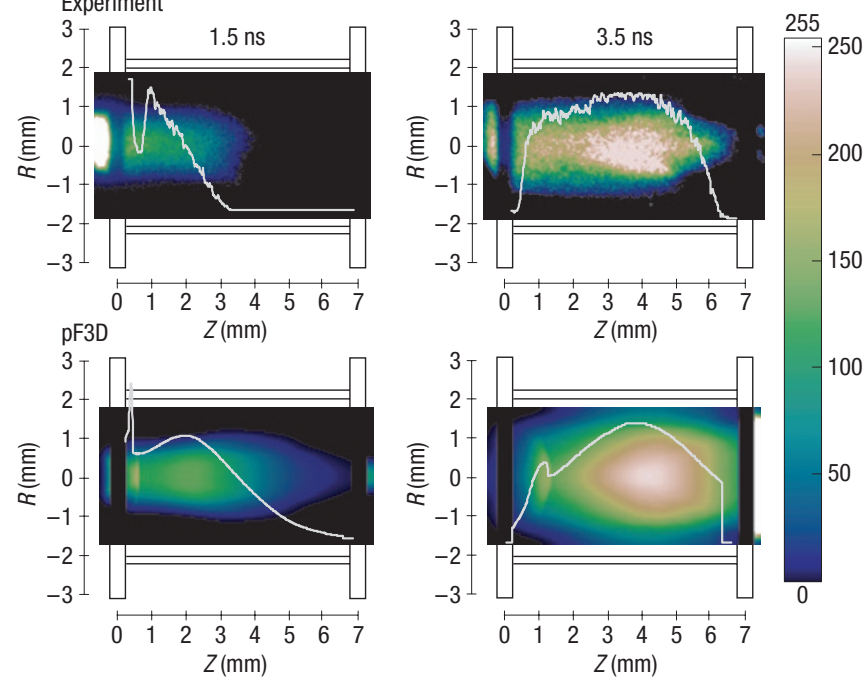

Figure 4 Measured and calculated X-ray propagation images at $3.5 \mathrm{keV}$ together with the relative intensity as a function of length (white curves). Experiments with full smoothing show efficient propagation close to optimum propagation as predicted by pF3D calculations. Beams only smoothed with CPP stall at $1.5 \mathrm{~ns}$ owing to filamentation.

beam-conditioning on laser propagation in large-scale plasmas. Although many outstanding problems remain in laser-plasma interaction physics and these experiments using $\mathrm{CO}_{2}$-filled targets produced too much backscatter to be a candidate for ignition designs, our ability to model laser-plasma experiments, where energy deposition, heat conduction, filamentation and SBS losses compete with different beam-conditioning options, enables the design of the next experiments planned with 96 beams. Modelling predicts that for future ignition experiments, reaching a higher electron temperature $\left(T_{\mathrm{e}}=5 \mathrm{keV}\right)$ and using a He-hydrogen gas mix to increase ion wave damping are key to controlling SBS over large-scale plasmas ${ }^{3-6,30}$, whereas polarization smoothing and SSD available on the NIF should ensure good propagation of the beams to reach the high- $Z$ hohlraum wall. 


\section{METHODS}

The measured suppression of filamentation with full laser beam smoothing can be understood by the reduction of the number of high-intensity speckles in the laser beam. On the NIF, $\mathrm{CPP}^{21,22}$ provide the laser spot size with super-gaussian profiles. A CPP splits the beam into independent overlapping beamlets that are randomly phased. Their interference creates a globally smooth speckle pattern in the focal plane.

Polarization smoothing ${ }^{19,20}$ divides the light power into two polarizations, each of which effectively has independent phases and thus an uncorrelated speckle pattern in the plasma. Thus, the beam contrast is instantaneously divided by $\sqrt{ } 2$ and the number of high intensity speckles is significantly reduced, with an effective intensity divided by 2 for the tail of the intensity distribution. $S_{S D}^{18}$ will result in a similar reduction, but in a time-averaged sense. With NIF SSD bandwidth, the beam contrast is reduced over timescales larger than 4 ps. Filamentation develops over hydrodynamic timescales larger than $t$. Adding polarization smoothing and SSD to CPP-smoothed beams results in a several orders of magnitude reduction in the likelihood of intense speckles with intensities of $\sim 5 I_{0}$ when compared with beams that apply only CPPs.

To resolve speckle-scale physics, the cell size of the pF3D mesh has to be of the order of the laser wavelength. A full 3D mesh covering the experimental images of Fig. 4 would then require half a trillion grid cells, with ten thousand time steps to simulate a $3.5 \mathrm{~ns}$ experiment. To reduce the computational problem to a more tractable size, a hybrid approach was developed in which the light propagation and hydrodynamics equations are solved in a 2D plane, whereas heat conduction and energy deposition are computed on a 3D coarser grid. We then use a multigrid iterative algorithm to solve the nonlinear diffusion equation describing heat conduction, with a source term calculated by rotating the $2 \mathrm{D}$ grid along the axis of propagation and renormalizing to account for $2 \mathrm{D}$ versus $3 \mathrm{D}$ divergence. Projection between different grid sizes is done by linear interpolation. Because the mean free path of electrons is typically hundreds of laser wavelengths, a refinement ratio of 32 was used, enabling simulations of the entire experiment.

\section{Received 12 0ctober 2006; accepted 2 August 2007; published 2 September 2007.}

\section{References}

1. Moses, E. I. \& Wuest, C. R. The National Ignition Facility: Laser performance and first experiments Fusion Sci. Technol. 47, 314-322 (2005).

2. Cavailler, C. Inertial fusion with the LMJ. Plasma Phys. Cont. Fusion 47, B389-B403 (2005)

3. MacGowan, B. J. et al. Laser plasma interactions in ignition-scale hohlraum plasmas. Phys. Plasmas 3 , 2029-2040 (1996)

4. Fernandez, J. C. et al. Measurements of laser-plasma instability relevant to ignition hohlraums. Phys. Plasmas 4, 1849-1856 (1997).

5. Niemann, C. et al. Intensity limits for propagation of 0.527 um laser beams through large-scale-length plasmas for inertial confinement fusion. Phys. Rev. Lett. 94, 085005 (2005).

6. Froula, D. H. et al. Ideal laser-beam propagation through high-temperature ignition hohlraum plasmas. Phys. Rev. Lett. 98, 085001 (2007).

7. Huller, S., Mounaix, Ph. \& Pesme, D. Numerical simulation of filamentation and its interplay with SBS in underdense plasmas. Phys. Scr. T 63, 151-157 (1996).
8. Schmitt, A. J. \& Afeyan, B. B. Time-dependent filamentation and stimulated Brillouin forward Schmitt, A. J. \& Afeyan, B. B. Time-dependent filamentation and stimulated Brillou
scattering in inertial confinement fusion plasmas. Phys. Plasmas 5, 503-517 (1997).

9. Myatt, J. et al. Modeling stimulated Brillouin scattering in the underdense corona of a direct drive inertial confinement fusion target. Phys. Plasmas 11, 3394-3403 (2004).

10. Berger, R. L. et al. On the dominant and subdominant behavior of stimulated Raman and Brillouin scattering driven by nonuniform laser beams. Phys Plasmas 5, 4337-4356 (1998).

11. Still, C. H. et al. Filamentation and forward Brillouin scatter of entire smoothed and aberrated laser beams. Phys. Plasmas 7, 2023-2032 (2000).

12. Lindl, J. D. et al. The physics basis for ignition using indirect-drive targets on the national ignition facility. Phys. Plasmas 11, 339-491 (2004).

13. Atzeni, S. \& Meyer-ter-Vehn, J. The Physics of Inertial Fusion. Beam Plasma Interaction, Hydrodynamics, Hot Dense Matter (Oxford Univ. Press, Oxford, 2004).

14. Glenzer, S. H. et al. Progress in large scale length laser plasma interactions. Nucl. Fusion 44, S185-S190 (2004).

15. Dewald, E. L. et al. First laser-plasma interaction and hohlraum experiments on the National Ignition Facility. Plasma Phys. Cont. Fusion 47, B405-B417 (2005).

16. Landen, O. L. et al. The first target experiments on the National Ignition Facility. J. Phys. D 133, 43-51 (2006)

17. Haynam, C. et al. National Ignition Facility laser performance status. Appl. Opt. 46, 3276-3303 (2007)

18. Skupsky, S. et al. Improved laser-beam uniformity using the angular-dispersion of frequency-modulated light. J. Appl. Phys. 66, 3456-3462 (1989).

19. Tsubakimoto, K. et al. Suppression of interference speckles produced by a random phase plate, using a polarization control plate. Opt. Commun. 91, 9-12 (1992).

20. Munro, D. H. et al. Polarization smoothing in a convergent beam. Appl. Opt. 43, 6639-6647 (2004)

21. Kato, Y. et al. Random phasing of high-power lasers for uniform target acceleration and plasm instability suppression. Phys. Rev. Lett. 53, 1057-1060 (1984).

22. Menapace, J. A. et al. Magnetorheological finishing for imprinting continuous phase plate structure onto optical surfaces. Proc. SPIE 5273, 220 (2003).

23. Spitzer, L. Jr \& Harm, L. Transport phenomena in a completely ionized gas. Phys. Rev. 89, 977-981 (1953).

24. Kilkenny, J. D. High-speed proximity focused x-ray cameras. Laser Part. Beams 9, 49-69 (1991).

25. Froula, D. et al. Full aperture backscatter measurements on the National Ignition Facility. Rev. Sci. Instrum. 75, 4168-4171 (2004).

26. Mackinnon, A. J. et al. Implementation of a near back-scattering imaging camera on the National Ignition Facility. Rev. Sci. Instrum. 75, 4183-4186 (2004).

27. Rozmus, W. et al. The local global analysis of the stimulated Brillouin-scattering in the regime of nonlinear sound waves. Phys. Fluids B 4, 576-593 (1992).

28. Heikkinen, J. A. et al. Ion-acoustic nonlinearities in stimulated Brillouin-scattering. Phys. Fluids 27 707-720 (1984)

29. Lefebvre, W. et al. Reduction of laser self-focusing in plasma by polarization smoothing. Phys. Plasmas 5, 2701-2705 (1998)

30. Meezan, N. et al. Role of hydrodynamics simulations in laser-plasma interaction predictive capability. Phys. Plasmas 14, 056304 (2007)

\section{Acknowledgements}

This work was carried out under the auspices of the US Department of Energy, National Nuclear Security Administration by the University of California, Lawrence Livermore National Laboratory under Contract No. W-7405-ENG-48. The authors would like to acknowledge the efforts of the engineering and operations staff at the National Ignition Facility.

Correspondence and requests for materials should be addressed to S.H.G.

\section{Author contributions}

The project was planned by S.H.G., L.D., B.A.H., O.L.L., B.J.M. and E.I.M. The experiment was designed by L.D., O.S.J., A.B.L., S.L., N.M., C.H.S., E.A.W. and L.J.S., and carried out by S.H.G., D.H.F., S.D., C.H., J.P.H., D.H.K., A.J.M., C.N. and B.K.F.Y. Target fabrication was carried out by R.J.W. The data were analysed by D.H.F., J.P.H. and C.N. and simulations were carried out by L.D., R.L.B., M.D. and J.A.H.

Competing financial interests

The authors declare no competing financial interests.

Reprints and permission information is available online at http://npg.nature.com/reprintsandpermissions/ 